\title{
Field driven recovery of the collective spin dynamics of the chiral soliton lattice $($ )
}

Cite as: Appl. Phys. Lett. 116, 012403 (2020); https://doi.org/10.1063/1.5131067

Submitted: 10 October 2019 . Accepted: 16 December 2019. Published Online: 02 January 2020

F. J. T. Goncalves (D), Y. Shimamoto (D), T. Sogo, G. W. Paterson (D), Y. Kousaka, and Y. Togawa

\section{COLLECTIONS}

EP This paper was selected as an Editor's Pick
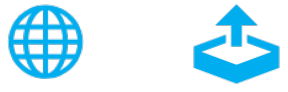

\section{ARTICLES YOU MAY BE INTERESTED IN}

Influence of electric polarization on Coulomb blockade in a super-paraelectric clusters assembly

Applied Physics Letters 115, 262901 (2019); https://doi.org/10.1063/1.5128846

Lateral straggling of implanted aluminum in $4 \mathrm{H}-\mathrm{SiC}$

Applied Physics Letters 116, 012101 (2020); https://doi.org/10.1063/1.5132616

Monolayer GaN excitonic deep ultraviolet light emitting diodes

Applied Physics Letters 116, 013101 (2020); https://doi.org/10.1063/1.5124828

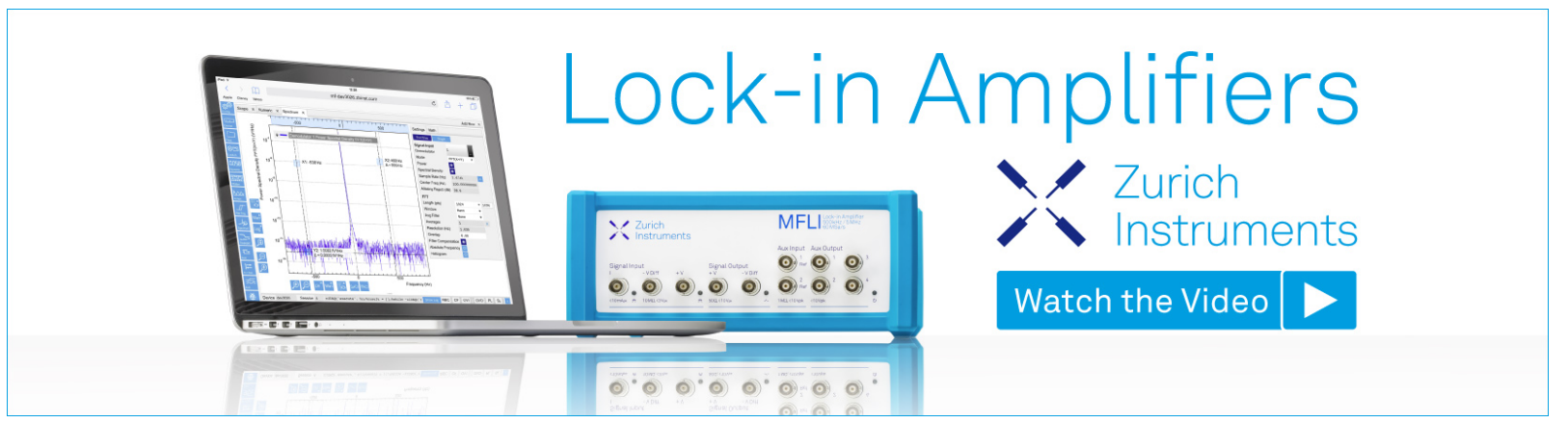




\title{
Field driven recovery of the collective spin dynamics of the chiral soliton lattice (ㅁ
}

Cite as: Appl. Phys. Lett. 116, 012403 (2020); doi: 10.1063/1.5131067

Submitted: 10 October 2019 . Accepted: 16 December 2019 .

Published Online: 2 January 2020

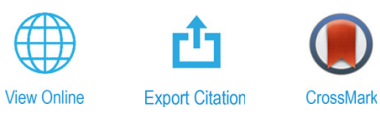

F. J. T. Goncalves, ${ }^{1, a)}$ (D) Y. Shimamoto, ${ }^{2}$ (D) T. Sogo, ${ }^{2}$ G. W. Paterson, ${ }^{3}$ (D) Y. Kousaka, ${ }^{2}$ and Y. Togawa ${ }^{2}$

\author{
AFFILIATIONS \\ ${ }^{\top}$ Helmholtz-Zentrum Dresden-Rossendorf, Institute of Ion Beam Physics and Materials Research, Bautzner Landstrasse 400, 01328 \\ Dresden, Germany \\ ${ }^{2}$ Department of Physics and Electronics, Osaka Prefecture University, 1-1 Gakuencho, Sakai, Osaka 599-8531, Japan \\ ${ }^{3}$ SUPA, School of Physics and Astronomy, University of Glasgow, Glasgow G12 8QQ, United Kingdom

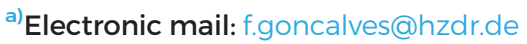

\begin{abstract}
We investigate the magnetic field dependence of the spin excitation spectra of the chiral soliton lattice (CSL) in the helimagnet $\mathrm{CrNb}_{3} \mathrm{~S}_{6}$, by means of microwave resonance spectroscopy. The CSL is a prototype of a noncollinear spin system that forms periodically over a macroscopic length scale. Following the field initialization of the CSL, we found three collective resonance modes over an exceptionally wide frequency range. Upon further reducing the magnetic field toward $0 \mathrm{~T}$, the spectral weight of these collective modes was disrupted by the emergence of additional resonances whose Kittel-like field dependence was linked to coexisting field polarized magnetic domains. The collective behavior at a macroscopic level was only recovered upon reaching the helical magnetic state at $0 \mathrm{~T}$. The magnetic history of this noncollinear spin system can be utilized to control microwave absorption, with potential use in magnon-driven devices.
\end{abstract}

Published by AIP Publishing. https://doi.org/10.1063/1.5131067

Recently, magnetic systems with noncollinear spin textures have attracted immense attention due to their ability to act as magnonic conduits with a programmable band structure. ${ }^{1-4}$ Naturally, good field stability and spatial coherence of the underlying noncollinear magnetic structure are essential requirements to ensure efficient propagation and manipulation of the magnonic signals. In this sense, chiral helimagnetic materials are very promising as the spatial coherence is built-in primarily due to an energy balance between the antisymmetric Dzyaloshinskii-Moriya (DM) interaction $^{5,6}$ and the symmetric exchange interaction, enabling spatially robust magnetic textures such as magnetic skyrmions $s^{4,7,8}$ and the chiral helical and the chiral spin soliton lattice phases. ${ }^{9}$ In chiral helimagnetic materials, periodically self-assembled magnetic elements emerge and can be tuned efficiently with an external magnetic field. When dealing with magnetic materials capable of hosting these "naturally" assembled spin textures, there is the need to assess the impact of magnetic disorder and defects as these can disrupt the spatial coherence and consequently affect the collective excitations and the propagation of spin waves.

In this Letter, we report the existence of three spin resonance regimes observed while varying the external magnetic field within the chiral soliton lattice (CSL) phase of a chiral helimagnetic crystal, CrNb3S6, with micrometer sized dimensions. In micrometer sized crystals as such, there exists an energy barrier that opposes an otherwise continuous transition from a field saturated phase to the CSL phase. ${ }^{10}$ During the field process of overcoming this energy barrier, magnetic disorder can appear in the form of magnetic dislocations, which then vanish at zero magnetic field. ${ }^{11}$ In our experimental results, the magnetic field range where each of the three distinct resonance regimes was observed is qualitatively coincident with that of the magnetic dislocations discussed in Ref. 11.

In the field regime that precedes the emergence of a significant number of magnetic dislocations, microwave spectroscopy experiments detected three resonance modes, concurrent with the onset of the CSL phase. The lowest order modes were detected at $14-20 \mathrm{GHz}$, while a higher order mode appeared at approximately twice this frequency. The multimode character of the spin excitation spectra is linked to the periodic nonlinear modulation of the moments forming the CSL and is reported here following its theoretical prediction. ${ }^{12-14}$ The overcoming of the energy barrier occurs with a further decrease in the field strength toward $0 \mathrm{~T}$. Here, we found two types of modes, which we ascribe to intrinsic CSL modes and ferromagnetic, Kittel-like modes, indicating the existence of a disordered CSL phase. Interestingly, upon switching the polarization of the external magnetic field through $0 \mathrm{~T}$, we found a clear transformation in the amplitude 
and field dependence of the resonance modes measured in the increasing field branch toward the critical field. The emergence of the helical state at $0 \mathrm{~T}$ triggered the extinction of the disordered phase, enabling the recovery of the collective spin excitation expected for an ideal, ordered CSL phase. Thus, we shed light on the differences between the spin excitation spectra of ordered and disordered CSL phases in micrometer sized crystals and reveal a magnetic field protocol that triggers only the spin wave resonance of the ordered CSL phase.

The spin configuration of the helimagnetic compound $\mathrm{CrNb}_{3} \mathrm{~S}_{6}$ at $0 \mathrm{~T}$ (below a critical temperature of $127 \mathrm{~K}$ ) corresponds to the chiral helical state consisting of $2 \pi$ magnetic kinks (MKs) arrayed with a periodicity of $48 \mathrm{~nm} .{ }^{6,15}$ Due to the symmetry of the crystal, two types of field-driven phase transitions can occur. When a magnetic field is applied parallel to the chiral helical axis, the helical state undergoes a transition to the saturated state via a conical phase. In this case, the spins cant toward the helical axis until the saturation is reached at a field magnitude of $2 \mathrm{~T} .{ }^{13}$ On the other hand, when a magnetic field is applied perpendicular to the helical axis, the chiral spin soliton lattice (CSL) emerges. ${ }^{15}$ The CSL is composed of MKs periodically distanced by regions with field polarized spins. The periodicity of the MKs increases with the strength of $H$ until a critical field, $H_{C}$, is reached. Above $H_{C}$, the field polarized (FP) phase is obtained. In this field configuration, the magnitude of $H_{C}$ varies between 0.15 and $0.24 \mathrm{~T}^{16-18}$ Several interesting features of the CSL such as spatial coherence, robustness with regard to the magnetic field, and discretized behavior can be found in the literature, both in the limits of small micrometer sized $^{16,19-22}$ and in bulk specimens. ${ }^{23,24}$

Bulk crystals of $\mathrm{CrNb}_{3} \mathrm{~S}_{6}$ were grown using a chemical vapor transport method. ${ }^{23,25}$ A micrometer sized rectangular specimen was cut from a bulk crystal using a focused ion beam technique and attached onto the signal line of a coplanar waveguide using tungsten. The length along the helical axis, the width parallel to $H$, and thickness of the specimen were $58.6,12.4$, and $2.6 \mu \mathrm{m}$, respectively. In the present experiments, the microwave field driving the spin precession was set perpendicular to the chiral axis (for details, see Sec. I in the supplementary material).

A coplanar-waveguide based microwave spectroscopy technique was employed to measure the spin excitation spectra via the forward transmission parameter $S_{21}$ as a function of the frequency and the magnetic field (see the supplementary material for details). The corrected magnitude and the field derivative of $S_{21}$ are referred to as $\Delta S$ and $\mathrm{d} \Delta \mathrm{S} / \mathrm{d} H$, respectively. In the experiments, the magnitude of the external field, $|H|$, was first decreased from large field values (well above $H_{C}$ ) to $0 \mathrm{~T}$ and then increased from $0 \mathrm{~T}$ up to large fields, and these are referred to as decreasing and increasing field sweeps, respectively.

Figure 1(a) shows the amplitude of $\Delta S$ obtained while varying the magnitude of $\mu_{0} \mathrm{H}$ from $-200 \mathrm{mT}$ to $200 \mathrm{mT}$, in field steps of $5 \mathrm{mT}$, at a temperature of $20 \mathrm{~K}$. In the field polarized (FP) phase, we identified three to four resonance modes with a Kittel-type field dependence, which is consistent with previous reports. ${ }^{26,27}$ At a magnetic field of $-138 \mathrm{mT}\left(H_{J}\right)$, the Kittel-like modes were replaced by three resonance branches whose field dependence is labeled as type-I modes. This transformation in resonance behavior marked the emergence of the CSL phase. As the field magnitude was decreased further, the resonance frequency of the two type-I modes at lower frequencies varied between 14 and $20 \mathrm{GHz}$, while the frequency of the third mode
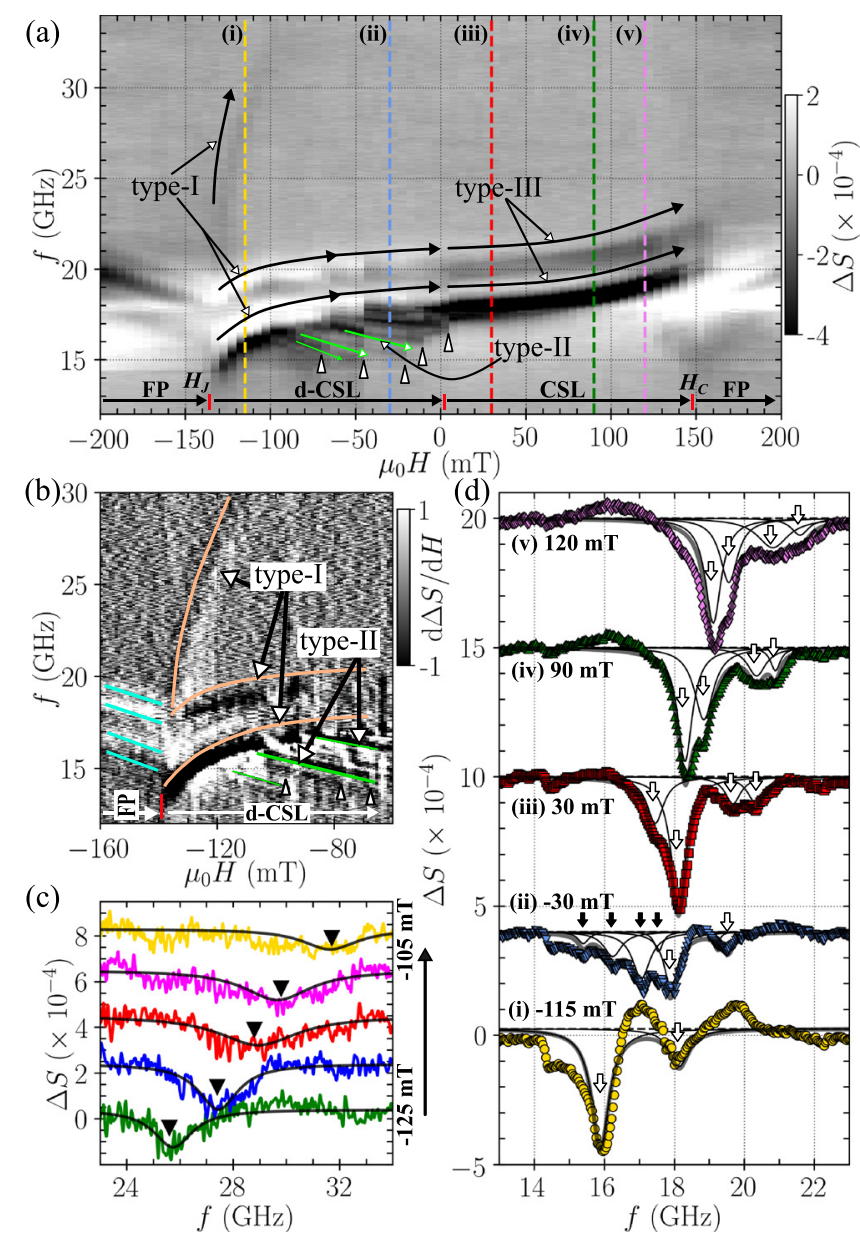

FIG. 1. (a) Amplitude of $\Delta S$ plotted as a function of frequency, $f$, and external magnetic field, $H$. The horizontal lines indicate the magnetic phases FP, disorderedCSL, and CSL, and their arrowheads indicate the field sweep direction. The elongated triangles displayed vertically indicate the fields where the number of type-II modes changed abruptly. (b) Plot of $d \Delta S / d H$ as a function of $f$ and $H$. Data obtained while sweeping $\mu_{0} H$ from $-200 \mathrm{mT}$ to $+200 \mathrm{mT}$ in field steps of $1 \mathrm{mT}$. (c) Line trace of the high order mode plotted as a function of $f$ in the vicinity of $H_{C}$. Markers indicate the center frequency of the resonance. (d) Amplitude profiles of $\Delta S$ at the various stages of the field sweep [dashed lines in (a)]. Open and full black arrows indicate the center frequency of the resonance modes of type-I/III and type-II modes, respectively. The line traces are Lorentzian fits to the various resonance modes.

increased rapidly from 18 to $35 \mathrm{GHz}$. Between $-138 \mathrm{mT}$ and $-100 \mathrm{mT}$, the type-I modes followed the outset of a domelike field dependence, which is consistent with the expected behavior in the intrinsic CSL phase. ${ }^{12,13}$ In order to clarify the field behavior of the type-I modes, we show the field derivative plot of $\Delta S$ in Fig. 1(b), which highlights the existence of three resonance modes. Figure 1(c) shows the amplitude profile of the resonance mode observed at high frequencies, for different values of $H$.

In the field range between $-100 \mathrm{mT}$ and $0 \mathrm{mT}$, in addition to the type-I modes, we observed a number of other resonance modes that clearly do not follow the field dependence intrinsic to the collective 
dynamics of the CSL. These are indicated in Figs. 1(a) and 1(b) as type-II modes. With the decreasing field magnitude toward $0 \mathrm{~T}$, each of these modes decreased linearly in frequency and disappeared suddenly at certain fields, as indicated by the triangular markers. In this field region, we observed five to six resonance modes, which vanished sequentially from lower to higher frequencies.

The switching of the field polarity, from small negative to positive fields $(-5 \rightarrow 5 \mathrm{mT})$, which expectedly enabled the appearance of the helical state, resulted in both the vanishing of the type-II modes and the sharp increase in the amplitude of the microwave absorption of the resonance modes identified as type-III in Fig. 1(a). The change in the resonance spectra can be visualized also in Fig. 1(d), which shows absorption lines measured at various magnetic field values before (i) and (ii) and after $0 \mathrm{~T}$ (iii)-(v), also corresponding to the vertical dashed lines in Fig. 1(a). Note that the frequency spreading of the resonance modes in Fig. 1(d-ii) is wider by $3 \mathrm{GHz}$ compared to (iii) due to the existence of the type-II modes indicated by the solid arrows, at lower frequencies.

The field dependence of the resonances is discussed further with reference to Fig. 2(a), which shows the absorption amplitude of the main type-I $(H<0)$ and type-III $(H>0)$ modes plotted as a function of $H$. We observed an increase in the amplitude of the main type-I mode between $-138 \mathrm{mT}$ and $-100 \mathrm{mT}$, followed by a decrease at $\mu_{0} H>-100 \mathrm{mT}$, ascribed to the emergence of the type-II modes. Above $0 \mathrm{~T}$, a drastic increase in the amplitude of the type-III modes was observed.

Despite the much larger resonance amplitude above $0 \mathrm{~T}$, the frequency of the two pairs of type-III modes seemed to connect smoothly across $0 \mathrm{~T}$ to the type-I modes observed in the decreasing field branch. However, at increasingly large values of $H$ in the increasing field process, the resonances deviated from the domelike field dependence of the type-I modes. Instead, the resonance frequency increased slowly at low fields and then more rapidly as $H$ approached $H_{C}$. Note that in the increasing field branch $(H>0)$, each broad resonance was composed of two overlapping modes with similar field dependences, as shown in Fig. 1(d). Importantly, the resonance frequency of the type-III modes followed a field dependence that is neither the intrinsic domelike CSL behavior of the type-I modes nor the Kittel-like dependence of the type-II modes observed in the decreasing field branch.
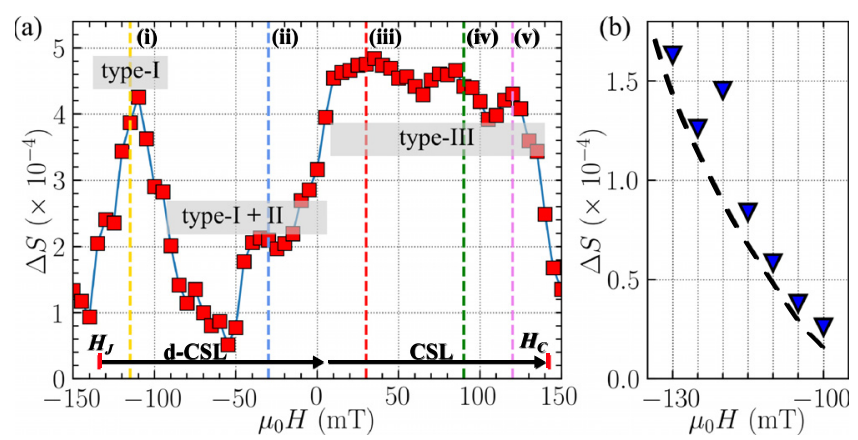

FIG. 2. (a) Absorption amplitude of the main resonance peak of type-I and type-III modes attributed to the CSL, plotted as a function of $H$. The colored lines mark the same fields as shown in Fig. 1. (b) Absorption amplitude of the high order mode as a function of $H$ in the vicinity of $H_{J}$ (dashed line is a guide to the eye).
In the resonance spectra observed near $H_{C} \sim 150 \mathrm{mT}$, we observed a field region, between $140 \mathrm{mT}$ and $160 \mathrm{mT}$, where the resonance modes attributed to the CSL and FP phases appear to coexist. We chose $H_{C}$ as the field magnitude above which the resonance attributed to the CSL decreased pronouncedly, while the Kittel-like resonances have clearly recovered. Well above $H_{C}$, the resonances exhibited a field dependence similar to that of the Kittel-like modes previously identified in the FP phase.

Previous experimental and theoretical results have shown that the collective spin dynamics of the CSL in finite size specimens depends strongly on the shape, the boundary conditions, and the intrinsic dynamics of the MKs. ${ }^{14,26,27}$ In Refs. 26 and 27, which focused on smaller specimens with a reduced number of MKs, the resonance behavior in the decreasing field process was characterized by a sharp frequency jump of typically $1.5-2.0 \mathrm{GHz}$, at $\mu_{0} H_{J} \sim 0.6 H_{C}$. This abrupt jump has been widely observed through various physical properties and has been recently attributed to the existence an energy barrier that controls the insertion of the MKs in the decreasing field process. ${ }^{10}$ The disordered phase was not unequivocally identified due to a more ordered phase transition and possibly due to a lower signal to noise ratio because of the sample size. In the present specimen, the abrupt frequency jump was not clearly observed. The absence of a clear jump in the resonance frequency and the fact that the magnitude of $H_{J}$ is comparable to $H_{C}$ appear to have promoted a near continuous formation of the CSL with decreasing $\mu_{0} H$ from $-138 \mathrm{mT}$ to $0 \mathrm{~T}$. Hence, a domelike field dependence on the type-I modes during the field decreasing process is observed. The domelike field dependence of the type-I resonance modes is consistent with the previous theoretical studies on the intrinsic excitation spectra of the CSL. Particularly in Ref. 12, where, in addition to the overall dome-shaped field dependence, it is discussed that the $n$th order CSL modes are dependent on a set of wavevectors linked to the spin modulation period of the MKs and that these modes can exist over a wide frequency range. Moreover, a rapid increase in the resonance frequency of the higher order modes and a simultaneous decrease in the mode amplitude are expected to occur with an increase in the density of MKs.

In the present Letter, the observation of the high order mode is an indication of the magnonic character of the CSL, which so far has only been discussed from the theoretical standpoint in Ref. 12. In particular, we confirmed that the resonance of at least one collective mode of the CSL increased markedly, from about $18 \mathrm{GHz}$ up to $35 \mathrm{GHz}$, and that the amplitude of the high order mode varied rapidly [see Fig. 2(b)] in the vicinity of $H_{J}$ (or $H_{C}$ ), where a considerable increase in the density of MKs is expected to occur. ${ }^{12}$

The field dependence of the resonances is discussed further with reference to Fig. 2(a), which shows the absorption amplitude of the main type-I $(H<0)$ and type-III $(H>0)$ modes plotted as a function of $H$. We observed an increase in the amplitude of the main type-I mode between $-138 \mathrm{mT}$ and $-100 \mathrm{mT}$, followed by a decrease at $\mu_{0} H>-100 \mathrm{mT}$, ascribed to the emergence of the type-II modes. Above $0 \mathrm{~T}$, a drastic increase in the amplitude of the type-III modes was observed.

The coexistence of the low order type-I modes with the Kittellike type-II modes strongly suggests that the CSL is mixed with regions where ferromagnetic alignment persists, possibly along with a large number of magnetic dislocations. The number and amplitude of typeII modes in the decreasing field process are linked to the spatial 
distribution and extent of the magnetic dislocations, which in turn reflect the contribution from the regions with ferromagnetic alignment. Presumably, such an overall disordered state was responsible for the partial disruption of the intrinsic collective resonance of the CSL, thereby preventing the CSL from acting as a collective entity at a macroscopic length scale.

The enhancement of the collective resonance behavior of the type-III modes $(H>0 \mathrm{~T})$ is believed to have been enabled by the expulsion of the magnetic dislocations.

So far, only spectroscopic techniques such as microwave resonance have been employed in the detection of the disordered CSL phase mainly due to the specimen thickness $(2.6 \mu \mathrm{m})$. However, recent studies of much thinner specimens $(\sim 100 \mathrm{~nm})$, using the Lorentz mode of transmission electron microscopy, showed the existence of magnetic edge dislocations, which are a signature of magnetic disorder in the CSL phase. It is important to note that, in a clear resemblance to the data discussed here, the magnetic dislocations were only observed while imaging in the decreasing field process. ${ }^{11}$

With changing the polarity of $H$ via zero magnetic field and the consequent emergence of the chiral helical state, the type-II modes vanished, triggering a different form of collective dynamics of the CSL. This is clearly seen through the increase in amplitude of the type-III modes above $0 \mathrm{~T}$ and the slope field dependence that differs from that observed in the type-I modes. In fact, a behavior similar to that of the type-III modes has been seen in previous experiments, ${ }^{26}$ where a slope field dependence was observed when following the same excitation configuration. The mechanism behind this slope field behavior is not clearly understood as more theoretical and experimental work is necessary on this front.

In light of the present experiments and recent theoretical considerations, ${ }^{28}$ one possible explanation might be that the collective resonance of the CSL became strongly dependent on the boundary spins due to the reordering of the spins near the surface (at $0 \mathrm{~T}$ ). While the type-I modes reflect the intrinsic, bulklike, response of the CSL, the type-III modes are a result of a collective CSL dynamics imposed by the boundary conditions that are ultimately dependent on the strength of the external field (hence the slope behavior). The absence of ferromagnetic domains and magnetic dislocations contributed to an increase in the absorption amplitude and an enhancement in the coupling between the microwave fields and the collective spin excitation modes of the CSL defined by the boundary spins. In the decreasing field process, the effect of the boundary spins on the collective CSL dynamics could have been suppressed due to the existence of a disordered phase.

In Fig. 3, we demonstrate the applicability and robustness of the resonance spectra corresponding to a magnetically ordered CSL phase at a macroscopic length scale. Once the disorder was removed from the specimen, the enhanced collective resonance of the ordered CSL phase persisted at all field values below $H_{C}$, regardless of which field sweep direction is adopted $[0 \rightarrow-200 \mathrm{mT}$ in (i) or $0 \rightarrow 200 \mathrm{mT}$ in (ii)]. Clearly, this enhanced response is symmetric with regard to the magnitude of $H$, which contrasts to the disrupted CSL dynamics obtained in the presence of magnetic disorder, as already presented in Fig. 1.

In the data shown in Fig. 1(a), the high order mode is not clearly identified in the increasing field branch due to loss in sensitivity on that experiment. However, the data presented in Fig. 3 show a high

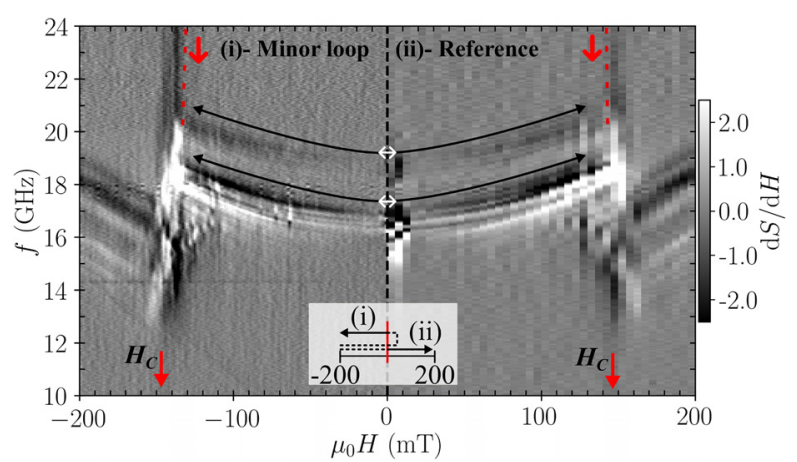

FIG. 3. Plot of $\mathrm{d} \Delta \mathrm{S} / \mathrm{d} H$ as a function of $f$ and $H$, corresponding to the increasing field branch of (i) a minor field loop and (ii) the increasing field branch obtained in a field sweep from $-200 \mathrm{mT}$ to $200 \mathrm{mT}$. The reversal field of minor loop (i) was $0.03 \mathrm{~T}$. Data obtained at $\mathrm{T}=50 \mathrm{~K}$. The horizontal arrows indicate the field sweep direction, and the inset illustrates the field process followed in (i) and (ii).

order resonance mode, as indicated by the red vertical arrows and dashed lines in both (i) and (ii) (see Sec. II in the supplementary material).

Following the qualitative interpretation above, the experimental results may be summarized in the following manner. A disordered CSL, composed of CSL and embedded ferromagnetic domains, produced two types of resonance modes with distinct and independent field behavior. As $H$ approached $0 \mathrm{~T}$, the resonance attributed to the ferromagnetic domains disappeared gradually until the helical phase was observed at $0 \mathrm{~T}$. At this point, a pronounced increase in the amplitude of the resonance modes was observed, suggesting that the ferromagnetic domains or the magnetic dislocations were disrupting the collective spin precession of the CSL. Once the helical phase was reached, the whole system resumed the collective behavior, which consisted of a number resonance modes with large amplitude and similar field behavior.

Importantly, we have demonstrated that the disordered state and inherent microwave resonance spectra can be completely erased and the collective dynamics restored simply by sweeping the external field through the helical magnetic phase at $0 \mathrm{~T}$. The control over the degree of magnetic disorder in the CSL, which was facilitated by the choice of the field process, can potentially enable the formation of field controlled channels of MKs that could serve as spin wave conduits.

See the supplementary material for further details on the experimental procedure, including a scanning electron microscope image of the specimen. Supplementary data on the origin and field robustness of the type-I and type-II modes are presented. In particular, data concerning a minor field loop that helped identifying the origin of the type-II modes are given. These data show the higher order type-I modes on both the decreasing and increasing magnetic field sweeps.

F.G. and Y.T. would like to thank T. Yuki for the support in the sample fabrication, A. Ovchinnikov, J. Kishine, and I. Proskurin for the discussions on this manuscript, and $\mathrm{K}$. Inoue and J. Akimitsu for promoting discussions on the topic of chirality. The authors acknowledge the support from the Grant-in-Aid for Scientific Research (Nos. 25220803, 17H02767, and 17H02923), 
Chirality Research Center (Crescent) in Hiroshima University. FG received support from JSPS International Research Fellowship No. 17F17316 and GWP received support from EPSRC Grant No. EP/ M024423/1.

\section{REFERENCES}

${ }^{\mathbf{1}}$ M. Krawczyk and D. Grundler, J. Phys.: Condens. Matter 26, 123202 (2014).

${ }^{2}$ C. S. Davies and V. V. Kruglyak, Low Temp. Phys. 41, 760 (2015).

${ }^{3}$ C. S. Davies, A. Francis, A. V. Sadovnikov, S. V. Chertopalov, M. T. Bryan, S. V. Grishin, D. A. Allwood, Y. P. Sharaevskii, S. A. Nikitov, and V. V. Kruglyak, Phys. Rev. B 92, 020408(R) (2015).

${ }^{4}$ T. Schwarze, J. Waizner, M. Garst, A. Bauer, I. Stasinopoulos, H. Berger, C. Pfleiderer, and D. Grundler, Nat. Mater. 14, 478 (2015).

${ }^{5}$ T. Moriya, Phys. Rev. 120, 91 (1960).

${ }^{6}$ T. Moriya and T. Miyadai, Solid State Commun. 42, 209 (1982).

${ }^{7}$ M. Garst, J. Waizner, and D. Grundler, J. Phys. D: Appl. Phys. 50, 293002 (2017).

${ }^{8}$ M. Weiler, A. Aqeel, M. Mostovoy, A. Leonov, S. Geprägs, R. Gross, H. Huebl, T. T. Palstra, and S. T. Goennenwein, Phys. Rev. Lett. 119, 237204 (2017).

${ }^{9}$ Y. Togawa, Y. Kousaka, K. Inoue, and J. Kishine, J. Phys. Soc. Jpn. 85, 112001 (2016).

${ }^{10}$ M. Shinozaki, Y. Masaki, R. Aoki, Y. Togawa, and Y. Kato, Phys. Rev. B 97, 214413 (2018).

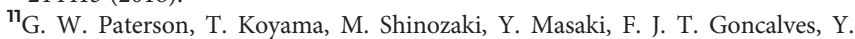
Shimamoto, T. Sogo, M. Nord, Y. Kousaka, Y. Kato, S. McVitie, and Y. Togawa, Phys. Rev. B 99, 224429 (2019).

12. Kishine and A. S. Ovchinnikov, Phys. Rev. B 79, 220405 (2009).

${ }^{13}$ J. Kishine and A. Ovchinnikov, in Solid State Physics, 1st ed. (Elsevier Inc., 2015), pp. 1-130.

${ }^{14}$ J. Kishine, I. Proskurin, I. G. Bostrem, A. S. Ovchinnikov, and V. E. Sinitsyn, Phys. Rev. B 93, 054403 (2016).

${ }^{15}$ Y. Togawa, T. Koyama, K. Takayanagi, S. Mori, Y. Kousaka, J. Akimitsu, S. Nishihara, K. Inoue, A. S. Ovchinnikov, and J. Kishine, Phys. Rev. Lett. 108, 107202 (2012).
${ }^{16}$ Y. Togawa, T. Koyama, Y. Nishimori, Y. Matsumoto, S. McVitie, D McGrouther, R. L. Stamps, Y. Kousaka, J. Akimitsu, S. Nishihara, K. Inoue, I. G. Bostrem, V. E. Sinitsyn, A. S. Ovchinnikov, and J. Kishine, Phys. Rev. B 92, 220412(R) (2015).

${ }^{17}$ K. Tsuruta, M. Mito, Y. Kousaka, J. Akimitsu, J. Kishine, Y. Togawa, and K. Inoue, J. Appl. Phys. 120, 143901 (2016).

${ }^{18}$ K. Tsuruta, M. Mito, Y. Kousaka, J. Akimitsu, J. Kishine, Y. Togawa, H. Ohsumi, and K. Inoue, J. Phys. Soc. Jpn. 85, 013707 (2016).

${ }^{19}$ J. Yonemura, Y. Shimamoto, T. Kida, D. Yoshizawa, Y. Kousaka, S. Nishihara, F. J. T. Goncalves, J. Akimitsu, K. Inoue, M. Hagiwara, and Y. Togawa, Phys. Rev. B 96, 184423 (2017).

${ }^{20}$ E. M. Clements, R. Das, L. Li, P. J. Lampen-Kelley, M.-H. Phan, V. Keppens, D. Mandrus, and H. Srikanth, Sci. Rep. 7, 6545 (2017).

${ }^{21}$ H. Han, L. Zhang, D. Sapkota, N. Hao, L. Ling, H. Du, L. Pi, C. Zhang, D. G. Mandrus, and Y. Zhang, Phys. Rev. B 96, 094439 (2017).

${ }^{22}$ M. Mito, H. Ohsumi, K. Tsuruta, Y. Kotani, T. Nakamura, Y. Togawa, M. Shinozaki, Y. Kato, J. Kishine, J. Ohe, Y. Kousaka, J. Akimitsu, and K. Inoue, Phys. Rev. B 97, 024408 (2018).

${ }^{23}$ T. Miyadai, K. Kikuchi, H. Kondo, S. Sakka, M. Arai, and Y. Ishikawa, J. Phys. Soc. Jpn. 52, 1394 (1983).

${ }^{24}$ Y. Togawa, Y. Kousaka, S. Nishihara, K. Inoue, J. Akimitsu, A. S. Ovchinnikov, and J. Kishine, Phys. Rev. Lett. 111, 197204 (2013).

${ }^{25}$ Y. Kousaka, Y. Nakao, J. Kishine, M. Akita, K. Inoue, and J. Akimitsu, Nucl. Instrum. Methods Phys. Res., Sect. A 600, 250 (2009).

${ }^{26}$ F. J. T. Goncalves, T. Sogo, Y. Shimamoto, Y. Kousaka, J. Akimitsu, S. Nishihara, K. Inoue, D. Yoshizawa, M. Hagiwara, M. Mito, R. L. Stamps, I. G. Bostrem, V. E. Sinitsyn, A. S. Ovchinnikov, J. Kishine, and Y. Togawa, Phys. Rev. B 95, 104415 (2017).

${ }^{27}$ F. J. T. Goncalves, T. Sogo, Y. Shimamoto, I. Proskurin, V. E. Sinitsyn, Y. Kousaka, I. G. Bostrem, J. Kishine, A. S. Ovchinnikov, and Y. Togawa, Phys. Rev. B 98, 144407 (2018).

${ }^{28}$ J. Kishine, V. E. Sinitsyn, I. G. Bostrem, I. Proskurin, F. J. T. Goncalves, Y. Togawa, and A. S. Ovchinnikov, Phys. Rev. B 100, 024411 (2019). 